\title{
Evidence for the shift of the glass transition near the particles in silica-filled elastomers
}

Julien Berriot*, Hélène Montes*, François Lequeux*\#, Didier Long ${ }^{+}$, Paul Sotta $^{+}$

*Physico-chimie des Macromolécules et des Milieux Dispersés

E.S.P.C.I 10 rue Vauquelin,75231 PARIS cedex 5 France

${ }^{+}$Laboratoire de Physique des Solides Bat. 510, Université Paris Sud, 91405 Orsay France

\# to whom correspondence should be addressed . email: francois.lequeux@espci.fr

Abstract : Filled elastomers exhibit complex dependence of their viscoelastic modulus, both as a function of temperature and frequency. Otherwise, recent observations on thin polymer films have shown that their glass transition temperature depends on their thickness. Based on these recent results, and on a recent model, we propose that the mechanical behaviour of the filled elastomer is strongly influenced by a gradient of the glass transition temperature in the vicinity of the particles. This allows us to suggest a specific temperature-frequency superposition law for filled rubbers. This law seems to apply very successfully on two systems with different dispersion qualities, revealing the existence of a glass transition temperature gradient in the vicinity of the particles.

\section{Introduction}

Filled elastomers are dispersions of solid particles in a polymeric network. They are commonly used in industry, but up to now, the origins of their mechanical properties are very unclear: all the previous analyses indicate that the properties of the polymer chains are 
strongly modified by the presence of solid particles. For instance, contrary to the pure elastomers, filled rubbers have precocious non-linear elastic responses known as the Payne effect [1]. They exhibit also important and sometime recoverable hysteresis when submitted to large deformations [2]. Moreover, their elastic modulus can even decreases with temperature while the one of the non reinforced matrix increases with temperature [3]. In this paper, we explain how the temperature dependence of the modulus can be interpreted in regards to the existence of a gradient of the glass transition temperature for the matrix in the vicinity of the interface with the particles.

More than 20 years ago, the existence of a glassy layer, or of a glassy interphase had already been suggested by some authors [4-5], for interpreting NMR measurements [6], and also some mechanical data [7-11]. However, the description has remained at a very qualitative level in particular, regarding the dependence on temperature of this layer, as well as regarding the underlying mechanism.

On the other hand, measurements of the glass transition temperature $T_{g}$ on polymer films by various authors and various methods have shown over the last few years that the glass transition of a polymer film depends on its thickness, and can either decrease or increase depending on the nature of the interaction between the film and its substrate [12-19]. This appears as a shift of the glass transition temperature near the interface. This shift is typically of order of $30 \mathrm{~K}$ for $10 \mathrm{~nm}$ thick films. Note that these are enormous effects when considering the viscosity of these films close to $\mathrm{Tg}$ and amount for the latter to an increase -for adsorbed films- or a decrease - for suspended films - by 3 or 4 orders of magnitude. Actually this shift can be interpreted as a gradient of the glass transition temperature induced by the interface [20]. The rigidity of the substrate - or its softness - can propagate over large distances in the sample leading to a dependence of the glass transition temperature with the distance to the interface. 
In this paper, we present mechanical results of filled elastomers. We interpret the results based on the concept of the dependence of the glass transition temperature with the distance to the interface. Our interpretation in terms of gradient of glass transition temperature is confirmed by both N.M.R. observations published in [21] and mechanical results. We finally claim that in filled rubbers, a gradient of the glass transition temperature is responsible for the temperature and frequency dependence of the viscoelastic modulus. This concept of gradient of glass transition temperature leads to a new specific temperature-frequency superposition. Finally we conclude that the mechanical behaviour of filled rubbers is controlled by spatial modification of the glass transition temperature similarly to thin polymer films.

The paper is organised as follows. We present the procedure used for the synthesis of our samples, and summarise N.M.R and structural measurements published elsewhere $[21,24]$. We then briefly describe mechanical results, which are however classical for these types of materials. We introduce the concept of the gradient of glass transition temperature. We explain how this concept allows to build a temperature-frequency superposition for filled rubbers. We have successfully tested this law for two sets of samples having different dispersion qualities. We finally discuss the role of this gradient on the viscoelastic modulus.

\section{Samples preparation and characterization}

We have prepared two types of polyethylacrylate samples filled with spherical silica particles. The particles are grafted either with 3-(trimethoxysilyl)propylmethacrylate (TPM) or with acetoxyethyl dimethylchlorosilane (ACS). The grafting density $\Gamma$ was checked by elemental analysis. The sample were prepared using the method developed by Ford [22]. The details of the sample preparation and analysis are given in [21, 24] 
Table I : Characteristics of the Reinforced Sample Sets and the Nonreinforced Elastomer Matrix

\begin{tabular}{|c|c|c|c|c|c|c|}
\hline Samples names & $\begin{array}{l}\text { Mean Silica } \\
\text { diameter } \\
(\mathrm{nm})^{1}\end{array}$ & $\begin{array}{c}\text { Grafting } \\
\text { Density } \\
\Gamma \\
\left(\mathrm{nm}^{-2}\right)\end{array}$ & $\begin{array}{l}\mathrm{e}_{\text {Graft }} \\
(\mathrm{nm})\end{array}$ & $\begin{array}{c}\text { Crosslinker } \\
\text { Concentration }{ }^{2} \\
(\%)\end{array}$ & $\begin{array}{c}\left\langle\mathrm{f}_{\mathrm{si}}^{\mathrm{N}}\right\rangle \\
\left(\mathrm{nm}^{-2}\right)^{3}\end{array}$ & $v_{\text {tot }} / v_{\mathrm{e}}^{3}$ \\
\hline EA & - & - & - & 0.3 & - & 1.58 \\
\hline ACS* & $54 \pm 7$ & $2 \pm 0.5$ & 0.3 & 0.3 & 0 & 1.58 \\
\hline TPM_I** & $50 \pm 7$ & $3.3 \pm 0.5$ & 0.7 & 0.3 & 1.1 & {$[1.66,1.89]^{4}$} \\
\hline
\end{tabular}

1 : from SANS measurements [22]. Dispersion state from SANS measurements * : presence of large aggregates; ** : there is the coexistence of any linear aggregates with single particles. 2 : per mole of ethylacrylate monomers. 3 : from ${ }^{1} \mathrm{H}$ NMR measurements [19], 4 : depending on the silica volume fraction.

In practice, silica particles of $50 \mathrm{~nm}$ diameter are prepared according to the classical Stöber method [23]. Their mean size and polydispersity were characterized by Small Angle Neutron Scattering (SANS) measurements which have shown that the particle surface are smooth [24]. The particles are first grafted in the initial Stöber solution. They are transferred by successive dialysis first in methanol, and then in ethylacrylate monomer. Lastly, a photosensitive initiator - Irgacure from CIBA- and a cross-linker - butanedioldiacrylate - are added, and polymerisation is done by U.V. illumination. For each silica volume fraction, the cross-linker concentration was kept equal to $0.3 \%$ per mol of acrylate monomers.

The chemical structure of the covering by the grafters molecules were analysed by ${ }^{29} \mathrm{Si}$ NMR measurements $[24,28]$. We observed that the TPM molecules form a dense shell around the particles. Indeed, after hydrolysis the TPM molecules have three hydroxyl groups which can form a covalent bond either at the silica surface or with other TPM molecules. A polycondensation between neighboring TPM molecules occurs leading to a dense structure at the particle surface. At the opposite, there is no polycondensation between ACS grafts due to their chemical structure. The ACS grafts form brushes over the particle surface. We can deduce the thickness $e_{\text {Graft }}$ of the grafting layer applying the relation 
$e_{\text {Graft }}=\frac{d}{2}\left(\sqrt[3]{1+\frac{6 \Gamma M_{m o l}^{G}}{d \rho^{G} N_{a}}}-1\right)$ where $\mathrm{M}^{\mathrm{G}}{ }_{\text {mol }}$ and $\rho^{\mathrm{G}}$ are the molar weight and the density of the graft molecules, $\mathrm{N}_{\mathrm{a}}$ the Avogadro number, $\Gamma$ the grafting density and $\mathrm{d}$ the particle diameter.

The interactions of the TPM and ACS grafters with the ethylacrylate monomers are different. The TPM coupling agent have one methacrylate group which can react with the acrylate monomers during the polymerisation step. TPM silica particles are then covalently bound to the acrylate matrix. On the contrary, the ACS coupling agents do not possess such methacrylate ending and they are not connected to the elastomeric network. This was confirmed by ${ }^{1} \mathrm{H}$ NMR measurements of the average density of topological constraints $v_{\text {tot }}$ [21] performed on each reinforced sample. We shown that $v_{\text {tot }}$ is the sum of three contributions : $v_{\text {tot }}=\left(v_{\mathrm{e}}+v_{\mathrm{c}}+v_{\mathrm{G}}\right)$. The first two contributions are due to entanglements $\left(v_{\mathrm{e}}\right)$ and to the crosslinker molecules $\left(v_{\mathrm{c}}\right)$ as in non reinforced elastomers. The third one $\left(v_{\mathrm{G}}\right)$ is proportional to both silica particle concentration and the grafting density $\Gamma$. This third contribution reveals the anchorage points between the particles and the elastomer matrix. The anchorage points introduced by the particles are localized at the particle surface. The particle functionality $\left\langle\mathrm{f}_{\mathrm{Si}^{\mathrm{i}}}{ }^{\mathrm{N}}\right\rangle$ was deduced for the two kinds of grafted silica particles. The characteristics for the both sets of filled elastomers are reported in table I.

Thus, we have obtained a polymeric network of cross-linked polyethylacrylate chains either connected (TPM-Silica) or not (ACS-silica) to particles. The elastic properties of the elastomer matrix located far from the particles have been probed by ${ }^{1} \mathrm{H}$ NMR and swelling measurements. We have especially analysed the influence of the anchorage points on the elastic properties of the elastomer matrix. From the NMR measurements we deduce that the crosslinker molecules react only with the acrylate monomers such that the crosslink density $v_{c}$ is not changed by the introduction of the grafted silica particles and is only determined by the 
initial cross-linker concentration $(0.3 \% \mathrm{~mol} / \mathrm{mol}$ of acrylate $)$ [21]. The swelling measurements have confirmed this result [29]. The anchorage points lead to a swelling restriction at the particle surface due to mechanical constraints but the swelling behaviour of the elastomer matrix located far from the particles is only determined by the cross-linker concentration [29]. Thus the morphology of the elastomer matrix does not depend nor on the presence of particles neither on the one of the grafter. We can thus deduce that far above Tg, the properties of the polymeric network in the bulk (i.e. far from the particles) are the same as those of the non reinforced matrix and are not modified by the filler concentration. Thus the elastic modulus of the matrix as well as its bulk glass transition temperature can be considered as independent of the filler concentration $[21,29]$. Moreover the cross-linker density is large enough so that we can consider that between two neighbouring particles the elastomer behave as a continuous elastic body.

Finally samples reinforced with TPM grafted silica particles - TPM_I samples- are optically transparent, and SANS measurements have revealed that at low q values the structure factor is proportional to $\mathrm{q}^{-0.7}$. the presence of few linear aggregates which coexist with individual particles [24]. On the contrary, samples filled with ACS silica particles - ACS samples- contain compact agglomerates [24].

\section{N.M.R. results}

We will first recall the results obtained by N.M.R. on TPM_I samples, that demonstrate the presence of a glassy layer with a temperature dependent thickness in our sample [21]. We have measured the transverse magnetisation relaxation in the filled system $\mathrm{M}_{\mathrm{fil}}(\mathrm{t}, \mathrm{T})$ of the protons belonging to the polymeric chains as a function of temperature $\mathrm{T}$. We have observed that $\mathrm{M}_{\mathrm{fil}}(\mathrm{t}, \mathrm{T})$ is a linear combination of the magnetization relaxation of the pure matrix at the same temperature and at a temperature below $\mathrm{T}_{\mathrm{g}}$ : 
$\mathrm{M}_{\mathrm{fil}}(\mathrm{t}, \mathrm{T})=\alpha \cdot \mathrm{M}_{\text {mat }}\left(\mathrm{t}, \mathrm{T}_{\mathrm{g}}-5 \mathrm{~K}\right)+(1-\alpha) \cdot \mathrm{M}_{\text {mat }}(\mathrm{t}, \mathrm{T})$

In NMR experiments, the signals from rigid - glassy - monomers and from mobile monomers are discriminated with respect to a typical frequency, which is given by the nuclear interactions involved in the NMR signal. The interaction relevant here is the dipolar broadening of the proton signal, which is of the order of $20 \mathrm{kHz}$. Thus the $\mathrm{T}_{\mathrm{g}}$ is determined here according to the WLF law extrapolated to the frequency $20 \mathrm{kHz}$.. This experimental result is observed for $\mathrm{T}>\mathrm{T}_{\mathrm{g}}+50 \mathrm{~K}$. It evidences the presence of a polymer shell around the particles exhibiting a glassy behaviour. Moreover the glassy fraction $\alpha$ decreases as temperature increases. This means that the thickness of the glassy polymer shell decreases with temperature as shown in figure 1. The method has been explained in more details in [21]. Note that similar results were observed previously by Kaufmann [6] on other filled elastomers. Contrary to the case of TPM_I samples, in the case of ACS sample we will not able to detect any significant contribution of a glassy shell to the N.M.R. signal.

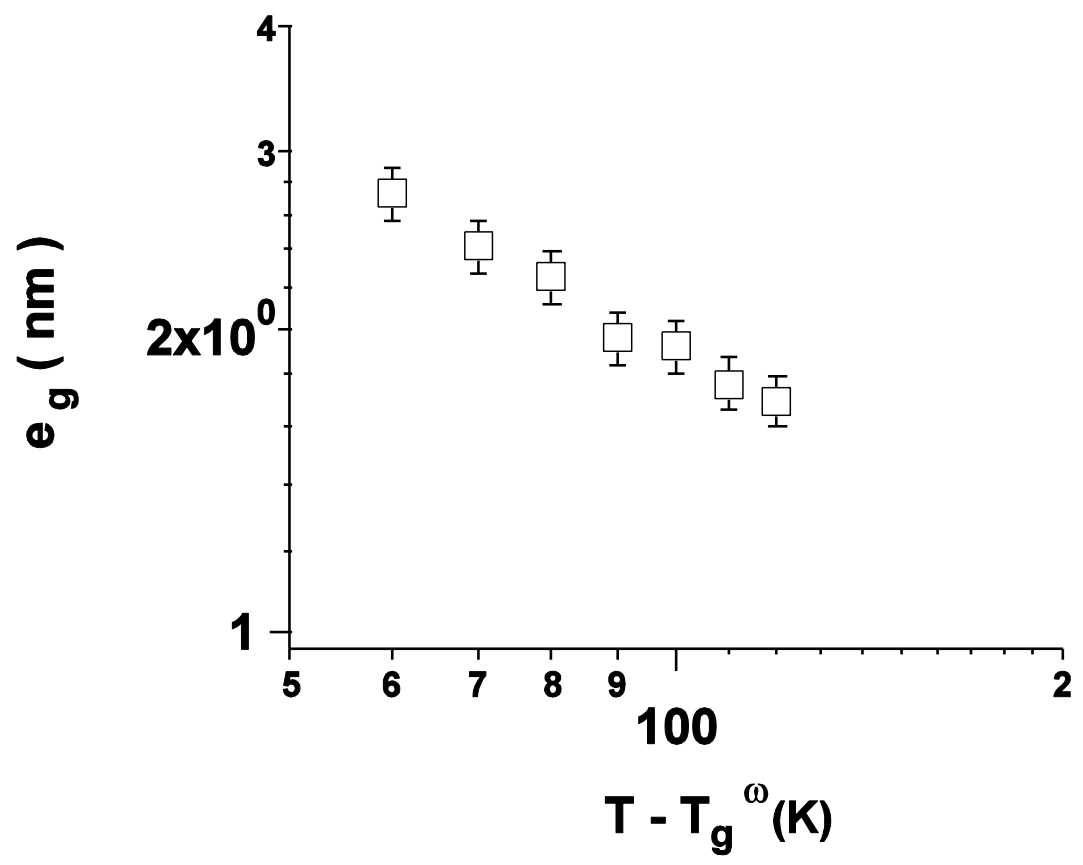

Figure 1: Variation of the thickness $\mathrm{e}_{\mathrm{g}}$ of the glassy shell around the particles versus temperature. Data from N.M.R measurements : 


\section{Mechanical measurements}

We have performed mechanical measurements far above $T_{g}$, on the rheometrics RDA II in simple shear flow with a plate-plate geometry. Samples were disks of $8 \mathrm{~mm}$ diameter and $2 \mathrm{~mm}$ thickness. They were glued with a cyanoacrylate glue (loctite) on the plates of the rheometer.

We have also performed mechanical measurements in the vicinity of $T_{g}$, with a Metravib viscoanalyzer. In that case, we have used sheets of dimension $2 \times 35 \times 7 \mathrm{~mm}^{3}$. The geometry was simple shear-torsion mode - and the sample were fixed with standard "clamps". For each sample we have measured both the elastic and the loss moduli as a function of frequency and temperature. We have always checked that we were in the linear regime. We have obtained the linear viscoelastic modulus in a broad range of temperature and frequency for the matrix and the filled samples of various concentrations.

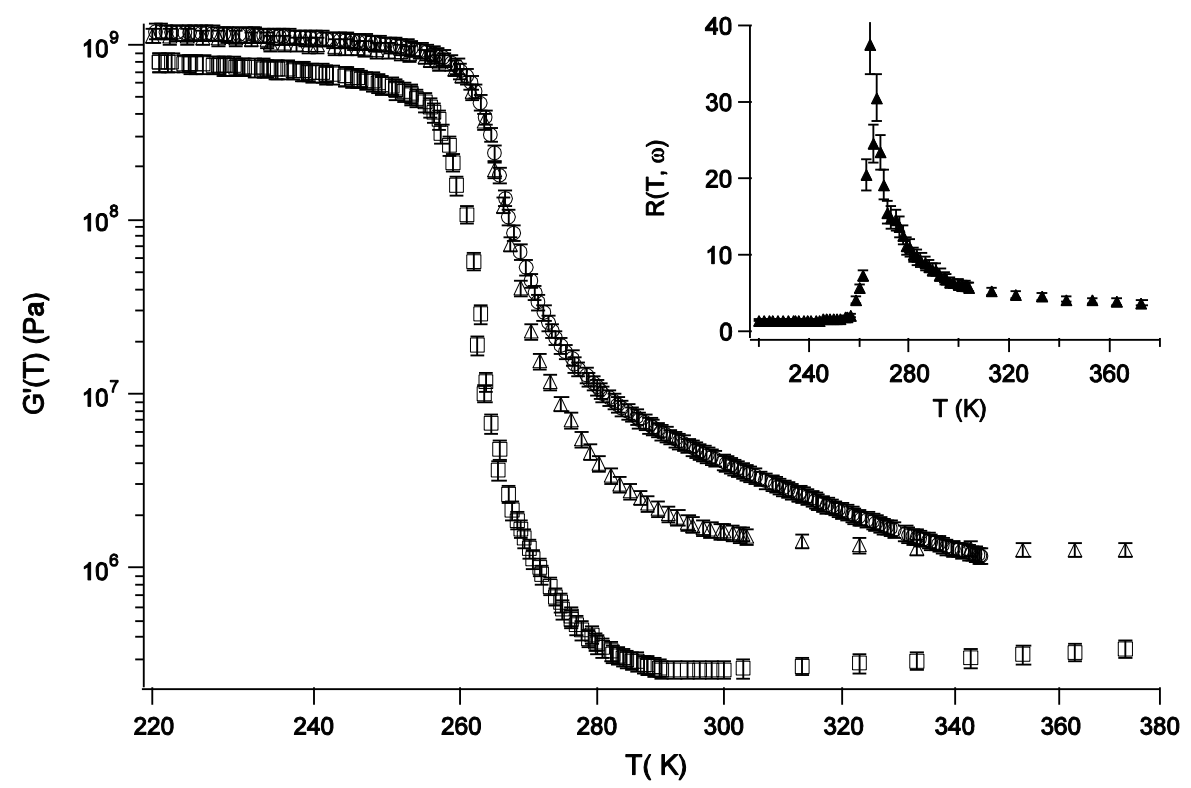

Figure 2 : Temperature dependence of the real part of the shear modulus $G^{\prime}(T)$ in the linear regime for different silica concentrations : $(\square): \Phi=0,(\triangle): \Phi=0.16$ TPM_I sample, (O): $\Phi=0.18$ ACS sample. Insert: Temperature dependence of the reinforcement for the TPM_I sample containing a silica volume fraction $\Phi=0.16$. 
The elastic modulus of the matrix, as plotted in figure 2 exhibits a plateau at low temperature, then a sharp decrease in the glass transition domain followed by a slight increase. These results are classical, and follow the usual WLF law in the glass transition domain. The sample presents a classical entropic behaviour at high temperature - the elastic modulus is proportional to the temperature and independent of frequency. In the following we will call $\mathrm{T}_{\mathrm{g}}{ }^{\omega}$ the temperature for which the loss modulus is maximum at the frequency $\omega$. We have measured this temperature $\mathrm{T}_{\mathrm{g}}{ }^{\omega}$ for frequencies varying from $10^{-2}$ to $10 \mathrm{~Hz}$. We found $\mathrm{T}_{\mathrm{g}}{ }^{\omega}=244 \mathrm{~K}$ at $\omega=0.01 \mathrm{~Hz}, \mathrm{~T}_{\mathrm{g}}{ }^{\omega}=248 \mathrm{~K}$ at $\omega=0.1 \mathrm{~Hz}, \mathrm{~T}_{\mathrm{g}}{ }^{\omega}=253 \mathrm{~K}$ at $\omega=1 \mathrm{~Hz}, \mathrm{~T}_{\mathrm{g}}{ }^{\omega}=257 \mathrm{~K}$ at $\omega=10$ Hz. We will use these values in the analysis of the data.

The results for TPM_I and ACS filler rubbers are qualitatively similar to the many other data obtained on various reinforced systems [4-5]. The elastic modulus exhibits a decrease in the glass transition domain which is broader than the pure matrix. This decrease for the samples described here - extends in the entropic regime of the matrix.

In Figure 3, we have reported for the TPM_I samples the temperature dependence of the elastic modulus for different silica concentration in the entropic regime of the matrix. It shows that for low silica volume fraction, the system exhibits an entropic regime at high temperature, similar to the one of the matrix. This entropic growth of the modulus progressively disappears as the concentration increases. 


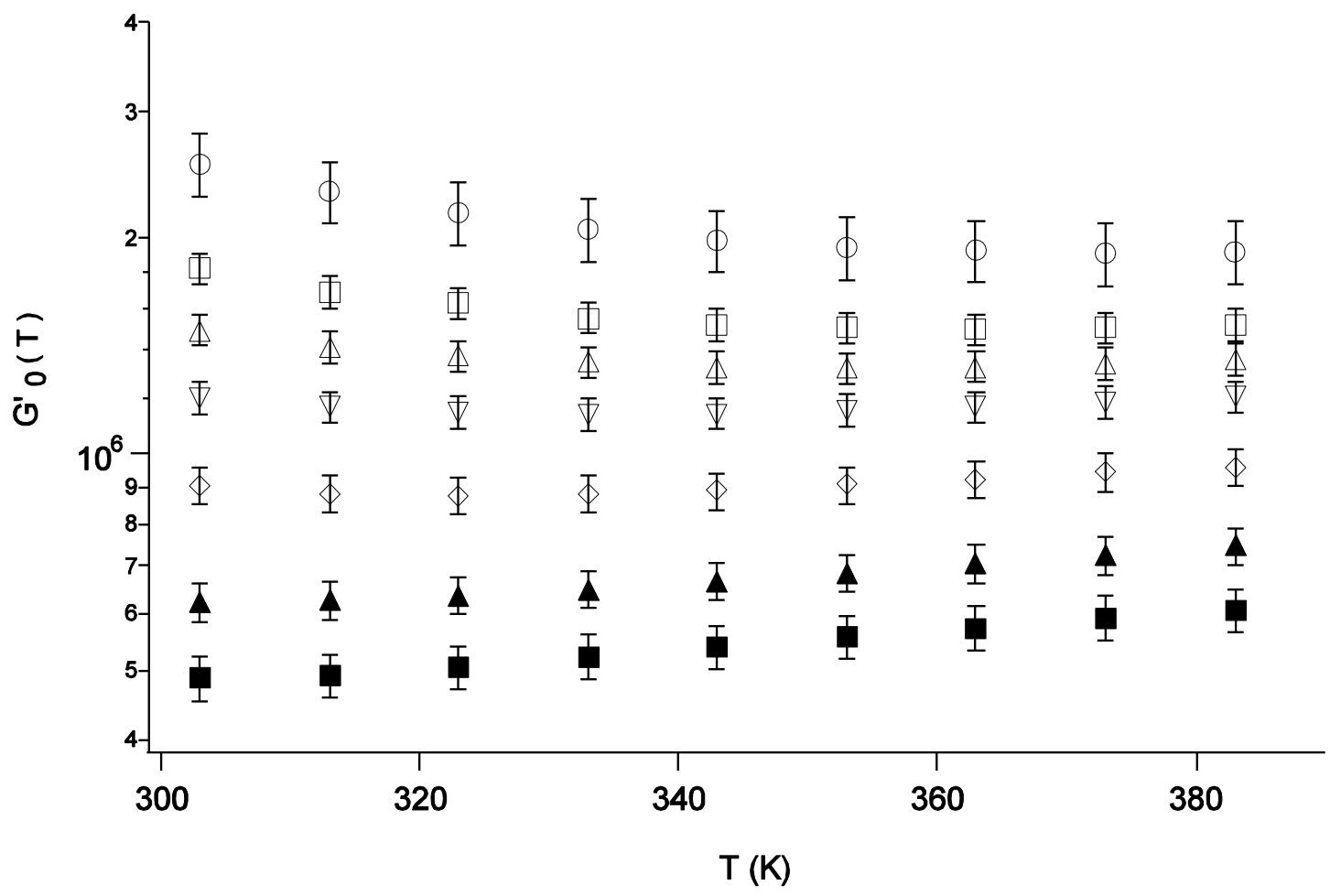

Figure 3 : Temperature dependence of the real part of the shear modulus $G^{\prime}(T)$ for TPM_I samples having varying silica concentration for temperatures $\mathbf{T}$ higher than $\mathbf{T}_{\mathrm{g}}{ }^{\omega}$ +50K. (ם) : $\Phi=0.07 ;(\Delta): \Phi=0.09 ;(\diamond): \Phi=0.12 ;(\nabla): \Phi=0.14 ;(\triangle): \Phi=0.15 ;(\square)$ : $\Phi=0.16 ;(O): \Phi=0.18$.

For the ACS sample, the decreases of the elastic modulus with temperature -see figure 2 - is extremely slow. We did not observe any entropic growth even for silica volume fraction as low as 0.07 .

One could think that the slow decrease of the elastic modulus with temperature corresponds simply to a broadening of the glass transition. In this case the classical timetemperature superposition law should apply for filled rubber, though with a different rescaling than for the polymer in the bulk. Let us recall that it consists in an horizontal shift - for the modulus as a function of the logarithm of the frequency- that superimposes the responses performed at various temperatures. Thus we have measured the frequency dependence of the shear modulus for various temperatures. In figure 4 , the elastic modulus is plotted for a volume fraction equal to $16 \%$ as a function of frequency and for various temperatures. The 
time-temperature superposition evidently fails for these systems - the slope of the elastic modulus with the logarithm of frequency clearly depends on the temperature.

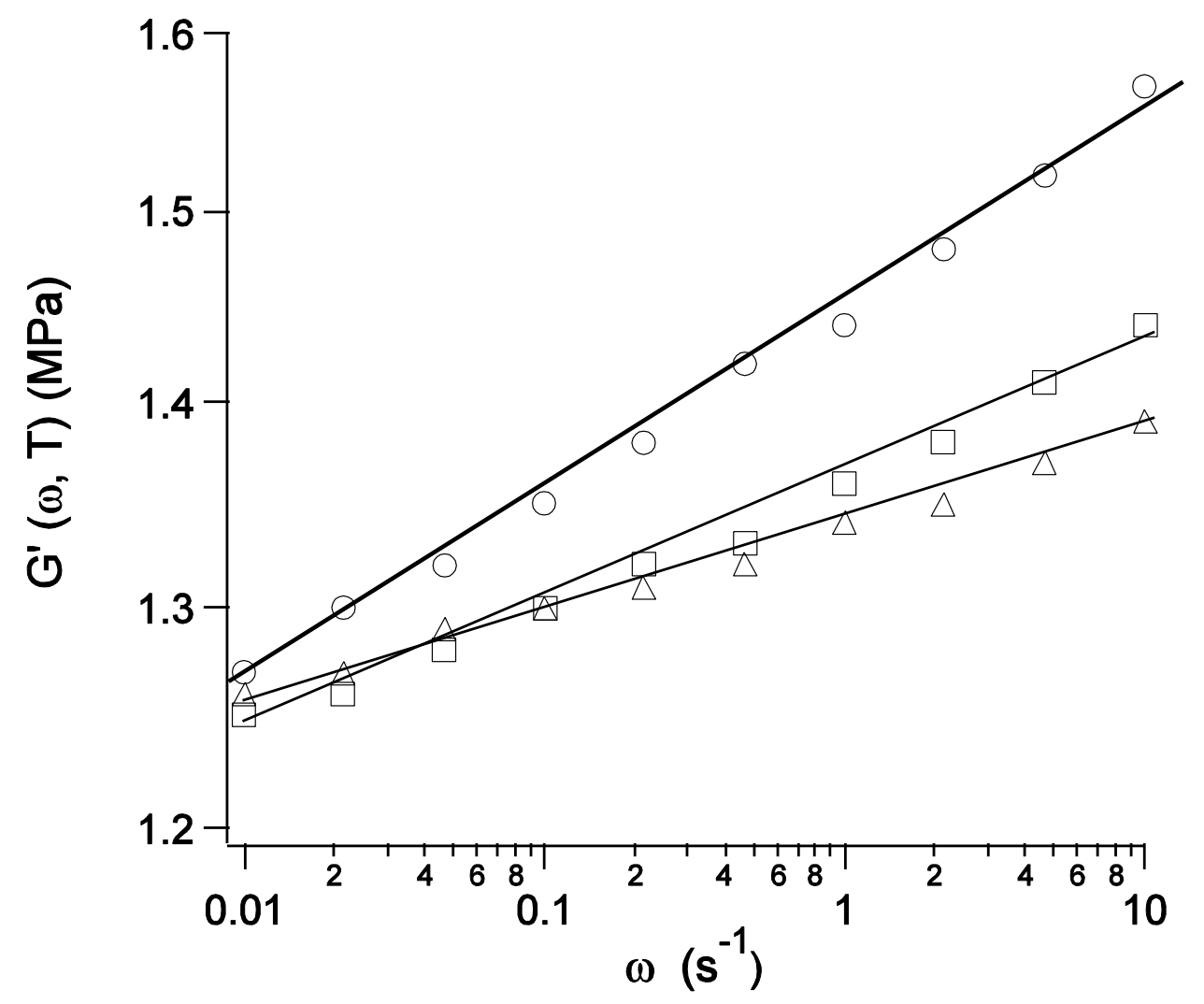

Figure 4 : Variation of the frequency dependence of the real part of the shear modulus with the temperature for a filled TPM_I elastomer with a silica volume fraction $\Phi=0.16$. (O): $\mathrm{T}=323 \mathrm{~K},(\square): \mathrm{T}=343 \mathrm{~K},(\triangle): \mathrm{T}=363 \mathrm{~K}$ The straight lines are eyes guide showing the variation of the temperature dependence of the slope of $G^{\prime}(\omega)$.

All these results, as well as all other results on other filled elastomers, show that this systems do not behave like a simple polymeric matrix containing solid particles. Indeed, in this case, the reinforcement $\mathrm{R}(\mathrm{T}, \Phi, \omega)$ defined as the ratio of the viscoelastic modulus $\mathrm{G}_{\mathrm{fil}}$ of the filled elastomer with $\mathrm{G}_{\mathrm{mat}}$ the one of the non reinforced polymer matrix would not depend on temperature and frequency for $\mathrm{T}>\mathrm{T}_{\mathrm{g}}{ }^{\omega}+50 \mathrm{~K}$ for each sample; it would be controlled only by the topological constraints density $v_{\text {tot }}$ and the particle volume fractions $\Phi$. 

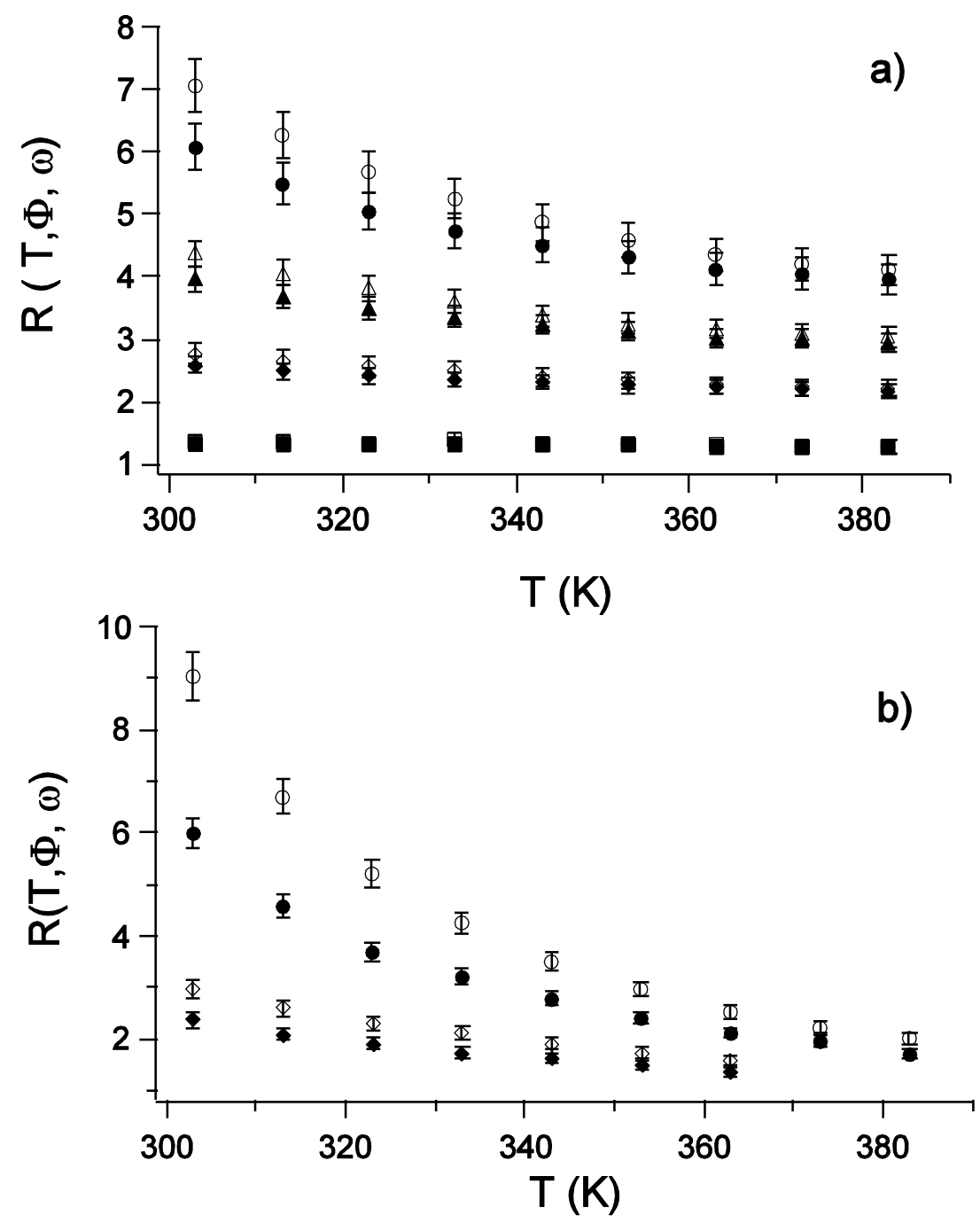

Figure 5 : Temperature and frequency dependence of the reinforcement $R(T, \Phi, \omega)$ for T> Tg $+50 \mathrm{~K}$ and two frequencies $\omega=1 \mathrm{~Hz}$ (open) and $\omega=0.01 \mathrm{~Hz}$ (filled). a) TPM_I samples; $(\square): \Phi=0.07$; $(\diamond): \Phi=0.12 ;(\triangle): \Phi=0.15 ;(O): \Phi=0.18$. b) ACS samples $(\diamond): \Phi=0.12 ;(O): \Phi=0.18$.

Figures $5 \mathrm{a}$ and $5 \mathrm{~b}$ clearly show that it is not the case here and that the reinforcement is both temperature and frequency dependent for temperatures higher than $\operatorname{Tg}^{\omega}+50 \mathrm{~K}$. Thus, the presence of solid particles modifies the dynamics of the chains in the matrix, as already noticed a few decade ago [4-5]. In order to account for these results, we have developed an approach based on recent results on the glass transition in thin polymer films. 


\section{Theoretical backgrounds : glass transition near a surface}

One of the main features of reinforced elastomers, is that many polymer chains are in contact with the particle surfaces. And it has been noticed that the surface of the fillers slows down the polymer chains dynamics $[5,21]$. A similar effect has been observed in details in thin polymer films coating surfaces $[12,13]$. More precisely, the glass transition temperature of a thin polymer film appears to be shifted as compared to the one of the bulk. This shift is positive for a polymer film strongly anchored on a solid substrate [17,18 ] and negative in other cases [12-16]. This shift varies typically as the inverse of the film thickness and is about $30 \mathrm{~K}$ for a thickness of $10 \mathrm{~nm}$. Recently, it has been proposed [20] that the surface induces a gradient of glass transition temperature in its vicinity. We will briefly resume the main concepts leading to this statement.

The basis of the model is the existence of strong dynamical heterogeneities observed in glassy polymers $[25,26]$. These heterogeneities lead to ratio of local dynamics of about 3 to 4 orders of magnitude over typical lengths of $2 \mathrm{~nm}$. The model describes explicitly these heterogeneities as originating from thermal fluctuations of density. And the heterogeneous nature of the dynamics originates from the high non-linear dependence of the dynamics with the density. Finally, the model [20] claims that the macroscopic dynamical properties of a glass is governed by those of a skeleton constituted by the slowest domains of the sample. Thus the dynamical properties of a glass should be controlled by the percolation of these slowest domains.

Otherwise, the percolation is modified by the dimension of the object. For instance, the percolation threshold for a 2-dimension object is lower than for a 3-dimension one. Thus one expects the dynamics to be modified in the vicinity of a surface. We will now precise how the percolation is modified near a surface. 
As the percolation corresponds to the connection of the slowest domains, it can be seen as the existence of infinite paths going only through slow domains. We will call these paths slow paths. Let us now consider a part of the sample located at a distance $z$ of an interface with a substrate. The dynamics, as compared to the bulk one, will be modified by the many slow paths emerging from the interface. If the substrate is rigid and the anchoring with the substrate strong enough, all the slow paths that emerge from the surface can be considered as slow. Thus the dynamics is slowed down near the surface. More precisely the minimum volume fraction necessary to constitute a skeleton is in this case smaller than the one for the bulk. This induces an increase of the glass transition temperature near the interface. On the opposite, if the substrate is not rigid - liquid or gaseous for instance - or if the anchoring is too weak, all the paths that would have been slow in a bulky sample, and that emerge from the interface are not anymore slow. Thus in that case, one expects that the dynamics near the interface is faster than the bulk one.

Thus the dynamics is modified near the surface because the slow skeleton that confers on the system its dynamics, is itself modified by the surface. Thus taking explicitly into account the dynamical fluctuations, the model gives the following results : the glass transition temperature $T_{g}^{\omega}(\mathrm{z})$ depends on the distance $\mathrm{z}$ from the surface according to :

$$
T_{g}^{\omega}(z)=T_{g}^{\omega}\left(1 \pm\left(\frac{\delta}{z}\right)^{1 / v}\right)
$$

where $T_{g}^{\omega}$ is the glass transition in the bulk at the frequency $\omega, \delta$ a length of the order of the nanometer, that depends on the anchorage at the interface and $v=.88$ the exponent for the correlation length in 3-dimensions percolation. The sign plus has to be considered in the case of an interface with a rigid body and a strong anchorage. The sign minus corresponds to an 
interface with a fluid body or with a rigid body and a weak anchorage. This result is in good agreement with the various experiments on thin films [12-19]. We will now apply this approach to filled elastomers.

Consequences for filled elastomers: establishment of a new $T-\omega$ superposition law.

Let us now consider the consequence of this effect on filled elastomers. We will limit ourselves to a positive shift of the glass transition near the particles, which is the case for our samples, as explained later on. We can use eq.(2) to estimate the properties of the polymer near the interface with the filler. A shift in glass transition will lead to spatial variations of the elastic modulus. Thus the elastic modulus of the matrix $G$ at position $\boldsymbol{x}$ will depend on the distance $z$ between the position $\boldsymbol{x}$ and the nearest interface, as expressed by :

$\mathrm{G}(\mathbf{x}, \mathrm{T}, \omega)=\mathrm{G}_{\text {mat }}\left(\mathrm{T}-\mathrm{T}_{\mathrm{g}}{ }^{\omega}\left(\frac{\delta}{\mathrm{z}(\mathbf{x})}\right)^{1 / v}, \omega\right)$

$\mathrm{G}_{\mathrm{mat}}\left(\mathrm{T}^{\prime}, \omega\right)$ is the modulus of the polymer matrix in the absence of filler, as a function of temperature $T^{\prime}$ and frequency $\omega$.

Thus, let us define the reinforcement $R$ as the ratio :

$$
R(T, \omega)=\frac{G_{\text {fil }}(T, \omega)}{G_{\text {mat }}(T, \omega)}
$$

of $\mathrm{G}_{\mathrm{fil}}$, the viscoelastic modulus of the filled elastomer, with $\mathrm{G}_{\mathrm{mat}}$ the one of the polymer without particles. In the absence of any modification of the dynamics of the polymer induced by the particles, the sample is composed by an homogeneous viscoelastic matrix containing solid inclusions. In that case, the reinforcement $\mathrm{R}$ is just a geometrical function which 
depends only on the respective position of the particles. So, for any given sample, $\mathrm{R}$ is independent of $\mathrm{T}$ and $\omega$. This statement holds whatever the spatial arrangement of the particles - even if the particles form aggregates. The fact that in most of filled rubbers the reinforcement $\mathrm{R}$ depends both on $\mathrm{T}$ and $\omega$ is a signature of the modifications of the dynamics of the polymer chains near the particle surfaces. In the frame of our assumption -glass transition temperature gradient in the vicinity of the particles - we will now introduce a temperature -frequency superposition law for filled rubbers. First let us go back to the local variation of the modulus described by equation (3)

Thus the difference between the room temperature and the local glass transition temperature changes of sign from the particle surface to the bulk matrix. Thus one expects a corresponding huge decrease of the elastic modulus from $10^{9} \mathrm{~Pa}$ to $10^{5} \mathrm{~Pa}$ as presented in figure 6 as a function of $\mathrm{z}$.

\section{$\log (\mathrm{G})$}

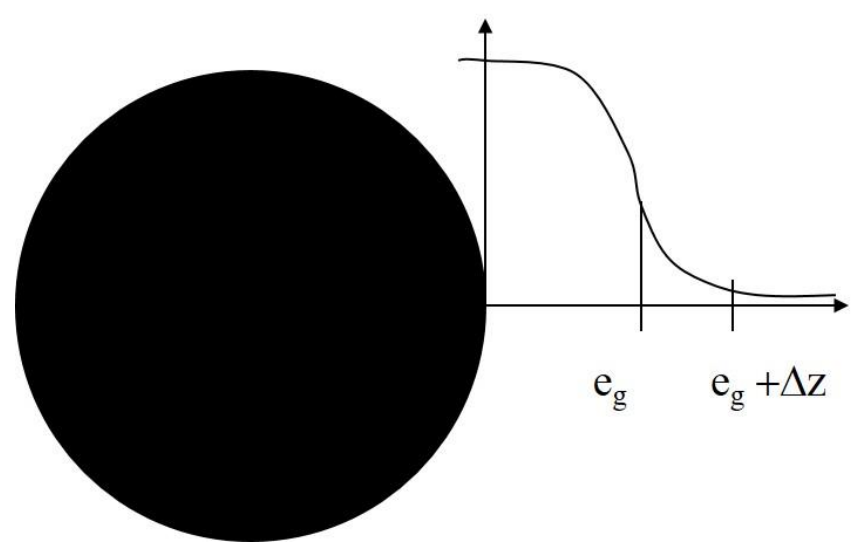

Figure 6 : Schematic representation of the variation of the modulus of the polymer chains near a particle (in black) versus the distance to the particle surface. For $z<\mathbf{e}_{\mathrm{g}}$, the polymer is in a glassy state $G=G_{\text {glass. }}$ At $z>e_{g}+\Delta z G=G_{N}$ where $G_{N}$ is the plateau modulus of the elastomer matrix.

We will first make a coarse grained approximation, assuming that the spatial variation of the modulus is extremely sharp. Thus the modulus can be considered as infinite for $\mathrm{z}<\mathrm{e}_{\mathrm{g}}$ 
and as equal to the one of the matrix for $\mathrm{z}>\mathrm{e}_{\mathrm{g}}$, where $\mathrm{e}_{\mathrm{g}}$ is the distance to the particle where the glass transition temperature is equal to the room one. Then $e_{g}$ can be seen as the thickness of the glassy shell surrounding the particles. It can be derived from equation (2) :

$$
e_{g}(T, \omega)=\delta\left(\frac{T_{g}{ }^{\omega}}{T-T_{g}{ }^{\omega}}\right)^{v}
$$

Here the frequency dependence of the glass transition temperature has been explicitly taken into account and leads to the variation of the thickness with frequency. However, for a given temperature $T$, it is always possible to find a frequency $\omega$ such that $e_{g}(T, \omega)$ is equal to a given thickness e. Thus in the frame of this coarse grained analysis, the reinforcement for a given thickness e is a geometrical function. It depends only on the respective positions of the particles and on the thickness e of their surrounding solid shell. Thus, as the thickness is a function of $\mathrm{T}_{\mathrm{g}}{ }^{\omega} /\left(\mathrm{T}-\mathrm{T}_{\mathrm{g}}{ }^{\omega}\right)$, we expect that for any given sample the reinforcement is a function only of :

$$
\mathrm{R}(\mathrm{T}, \omega) \cong \mathrm{R}\left(\frac{\mathrm{T}-\mathrm{T}_{\mathrm{g}}{ }^{\omega}}{\mathrm{T}_{\mathrm{g}}{ }^{\omega}}\right)
$$

where $\mathrm{R}$ is a function that varies from sample to sample.

Let us now generalize this frequency-temperature law to systems with any dispersion state, including the ones where particles can be bridged by their glassy shell. In that case, the gradient of glass transition, at a given position in the sample, can originate from the surfaces of more than one particle. Thus the expression (5) will no longer be valid as we have to include the influence of all the surrounding particles. However, it is then easy to show taking account in (2) and (3) of the contribution of many particles- that whatever the complexity of the particles arrangement, the position of the interface between molten and glassy polymer is a function only of $\mathrm{T}_{\mathrm{g}}{ }^{\omega} /\left(\mathrm{T}-\mathrm{T}_{\mathrm{g}}{ }^{\omega}\right)$. Thus equation (6) applies whatever the 
dispersion quality. But of course the absolute value of the function R depends each sample, and varies with the dispersion state.

This is the temperature-frequency superposition law that we suggest for filled elastomers. If the dependence of $\mathrm{R}$ in $\mathrm{T}_{\mathrm{g}}{ }^{\omega} /\left(\mathrm{T}-\mathrm{T}_{\mathrm{g}}{ }^{\omega}\right)$ is expected to depend dramatically with the volume fraction and the state of dispersion of the particles, this law is expected to apply for every sample, whatever the complex arrangement of the particles and their glassy shells including the presence of aggregates resulting from particles connected by glassy bridges. Effectively, whatever the structure of the glassy bridges and particles, it will not evolve if the temperature and the frequency are shifted such that $\mathrm{T}_{\mathrm{g}}{ }^{\omega} /\left(\mathrm{T}-\mathrm{T}_{\mathrm{g}}{ }^{\omega}\right)$ remains constant. Let's note that the modulus of the matrix increases with temperature at high temperature - it is the wellknown entropic behaviour. By dividing the modulus of the filled elastomer by the one of the non reinforced matrix, we take into account this effect.

According to this superposition law, the reinforcement exhibited by a given sample at various frequencies and temperature may collapse on a single curve if plotted as a function of $\mathrm{T}_{\mathrm{g}}{ }^{\omega} /\left(\mathrm{T}-\mathrm{T}_{\mathrm{g}}{ }^{\omega}\right)$ Before checking the validity of this law on various systems, we will now discuss the limitation of this law, which is only valid in the frame of our coarse-grained approximation

\section{Limitation of the time-temperature superposition law for filled rubber}

Let us first recall the time-superposition law for glassy polymer systems. This law deals with a precise situation : the viscoelastic modulus as a function of frequency at a given temperature, is equal to the one at another temperature, but for frequencies multiplied by a constant shift factor. On the opposite the elastic moduli of a polymer measured as a function of temperature at two different frequencies do not superimpose by a constant shift in temperature. This is discussed in details in [27]. 
Let us now go back to the viscoelastic modulus of the polymer in a filled rubber. When a measurement is performed, all the matrix is submitted to an oscillatory strain at the same frequency but its glass transition temperature varies. As explained above changing the frequency does not correspond to a constant temperature shift for the polymer in the whole sample from place to place. Thus the distribution of elastic modulus in the sample is not homogeneously shifted by a frequency change. Thus the gradient of the elastic modulus near $\mathrm{e}_{\mathrm{g}}(\mathrm{T}, \omega)$ depends on the temperature and frequency of the measurement even if the thickness of the glassy layer $\mathrm{e}_{\mathrm{g}}(\mathrm{T}, \omega)$ is kept constant. In other words, our temperature-frequency superposition law for filled rubber requires the coarse grained approximation. In the case where the elastic modulus varies smoothly from place to place, no superposition law could apply.

More precisely, this coarse-grained approximation is valid only if the domain where the elastic modulus goes from its glassy value to its rubbery one is small compared to the distance between neighbouring particles. This intermediate domain corresponds roughly to a variation in the shift in glass transition temperature of the order of $\Delta \mathrm{T} \approx 20 \mathrm{~K}$.

Thus differentiating equation (5), we get a thickness $\Delta \mathrm{z}$ for this intermediate domain of the order of :

$$
\Delta \mathrm{z} \approx \frac{\Delta \mathrm{T}}{\mathrm{T}_{\mathrm{g}}{ }^{\omega}} \frac{\mathrm{e}_{\mathrm{g}}^{1+1 / v}}{\delta^{1 / v}}
$$

The coarse-grained approximation will be valid as long as $\Delta \mathrm{z}$ remains smaller than characteristic distances of the elastic problem, typically the particle diameter. For diameter of $50 \mathrm{~nm}$, it requires $\Delta \mathrm{z}$ smaller than $5 \mathrm{~nm}$. This corresponds to a glassy thickness of $7.5 \mathrm{~nm}$, and thus to a value of $\left(\mathrm{T}-\mathrm{T}_{\mathrm{g}}{ }^{\omega}\right) / \mathrm{T}_{\mathrm{g}}{ }^{\omega}$ of 0.1 . Thus, we expect our temperature frequency superposition law for filled rubber to be valid only for of $\left(\mathrm{T}-\mathrm{T}_{\mathrm{g}}{ }^{\omega}\right) / \mathrm{T}_{\mathrm{g}}{ }^{\omega}$ larger than 0.1 . We will come back to this point later. 


\section{Rescaling our data}

We have plotted on figure $7 \mathrm{a}$ - respectively $7 \mathrm{~b}$ - the reinforcement $\mathrm{R}(\mathrm{T}, \omega)$ of the TPM_I and ACS samples as a function of $\mathrm{T}_{\mathrm{g}}{ }^{\omega} /\left(\mathrm{T}-\mathrm{T}_{\mathrm{g}}{ }^{\omega}\right)$ - for various silica concentrations, and for frequencies and temperatures varying respectively between from $10^{-2}$ to $10 \mathrm{~Hz}$, and from 30 to $100^{\circ} \mathrm{C}$. We see figure 7a that, in agreement with relation (9) all the data collapse on a single curve for each concentration for the TPM_I samples. The reinforcement $\mathrm{R}$ is always an increasing function of $\mathrm{T}_{\mathrm{g}}{ }^{\omega} /\left(\mathrm{T}-\mathrm{T}_{\mathrm{g}}{ }^{\omega}\right)$. Moreover, $\mathrm{R}$ increases with silica particles volume fraction and its slope versus the reduced variable increases also. 

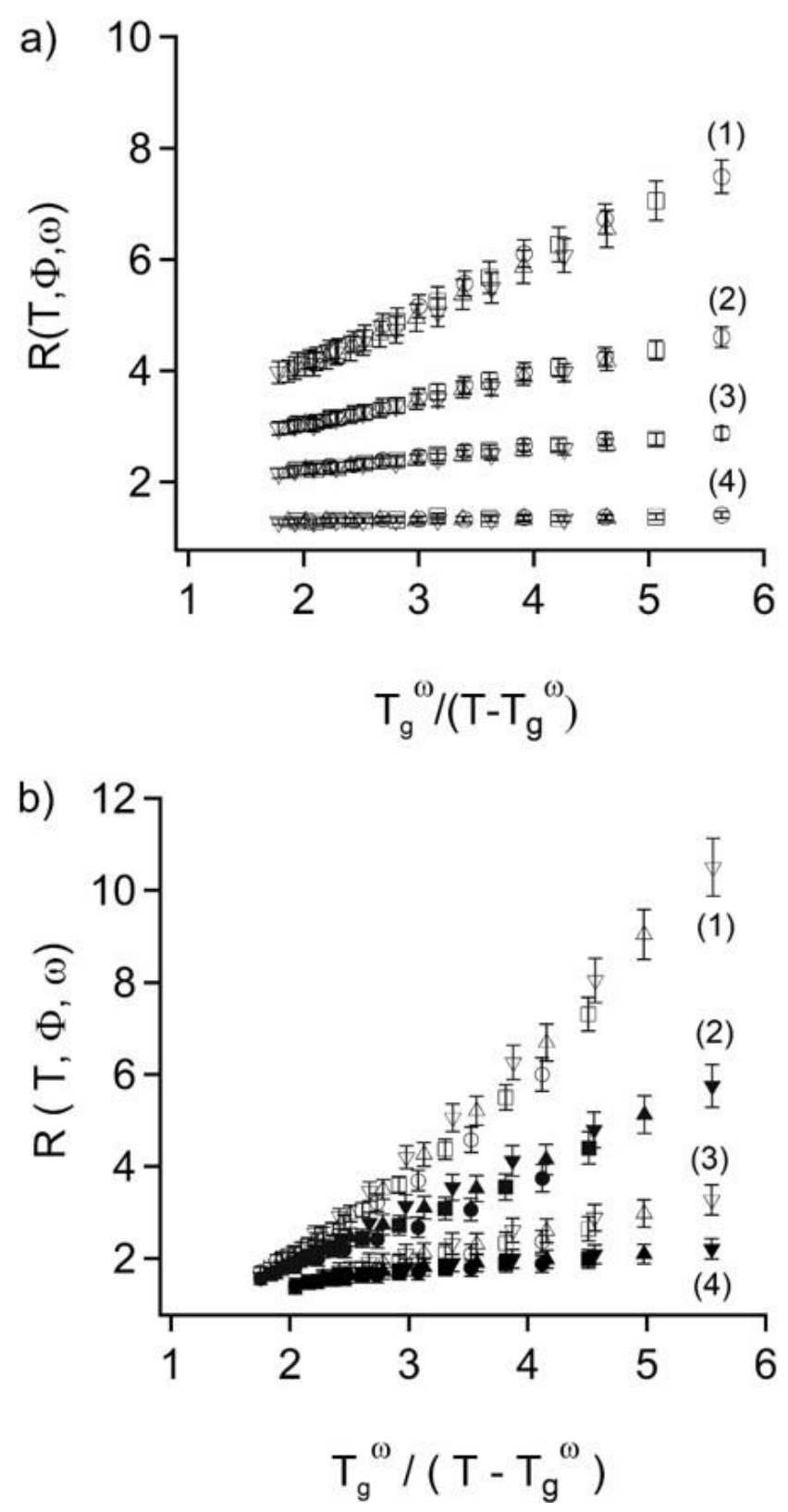

Figure 7 : a) Variation of the reinforcement $R$ versus $T_{g}{ }^{\omega} /\left(T-T_{g}{ }^{\omega}\right)$ for different frequencies and different silica concentrations for the TPM_I sample : (O) : $\omega=10 \mathrm{~Hz}$, ( $\square): \omega=1 \mathrm{~Hz},(\triangle): \omega=0.1 \mathrm{~Hz},(\nabla): \omega=0.01 \mathrm{~Hz} .(1): \Phi=0.18 ;(2): \Phi=0.15 ;(3): \Phi=0.12$; (4) : $\Phi=0.08$. b) Variation of the reinforcement $R$ versus $T_{g}{ }^{\omega} /\left(T-T_{g}{ }^{\omega}\right)$ for different frequencies and different silica concentrations for the ACS sample : for (1) : $\Phi=0.18$ and (3) : $\Phi=0.12(O): \omega=10 \mathrm{~Hz},(\square): \omega=1 \mathrm{~Hz},(\triangle): \omega=0.1 \mathrm{~Hz},(\nabla): \omega=0.01 \mathrm{~Hz}$. For (2) : $\Phi=0.15$ and (4) : $\Phi=0.09,(\bigcirc): \omega=10 \mathrm{~Hz},(\square): \omega=1 \mathrm{~Hz},(\Delta): \omega=0.1 \mathrm{~Hz},(\nabla)$ : $\omega=0.01 \mathrm{~Hz}$. 
This behaviour is similar for the ACS sample as shown on figure $7 \mathrm{~b}$. Both the value of $\mathrm{R}$ and its slope increases with the filler volume fraction. Moreover the slope of $\mathrm{R}$ versus $\mathrm{Tg}_{\mathrm{g}}{ }^{\omega}$ $/\left(\mathrm{T}-\mathrm{T}_{\mathrm{g}}{ }^{\omega}\right)$ is larger for the ACS samples than for the TPM_I one at similar volume fraction in particles. Otherwise the superposition for ACS samples is less satisfactory than for TPM_I samples and fails for $\mathrm{T}_{\mathrm{g}}{ }^{\omega} /\left(\mathrm{T}-\mathrm{T}_{\mathrm{g}}{ }^{\omega}\right)$ larger than 3.5. Thus despite these differences between the two systems, the frequency-temperature superposition that we have suggest is very satisfactory.

\section{Discussion}

Let us now discuss our results. At first the reinforcement $\mathrm{R}$ is not a constant but depends both on frequency and on temperature. This fact - as explained above - proves that the polymer dynamics is modified by the particles, and more precisely that the viscoelastic modulus is no longer homogeneous in the sample. Moreover, as R is an increasing function of $\mathrm{T}_{\mathrm{g}}{ }^{\omega} /\left(\mathrm{T}-\mathrm{T}_{\mathrm{g}}{ }^{\omega}\right)$ for both our samples in the temperature domain considered here - which corresponds to temperature above $\mathrm{Tg}+50 \mathrm{~K}$ - the dynamics is slowed down by the particles. This is consistent with N.M.R. results that exhibit the existence of a glassy shell for the TPM_I samples. Moreover N.M.R. results show that the glassy layer thickness decreases with temperature in agreement with eq. 5. as shown in figure 1 . This observation is reminiscent of the results obtained for thin polymers films [12-19]. Furthermore, as explained above, the assumption that the particles induce a gradient of glass transition temperature in their vicinity, leads to a temperature-frequency superposition, which is validated by our experimental data. This is actually the main results of our paper.

More precisely, for TPM_I samples containing only few small aggregates [24], the rescaling is very satisfactory for $\mathrm{T}-\mathrm{T}_{\mathrm{g}}{ }^{\omega}$ larger than $20 \mathrm{~K}$. 
But for ACS samples that contain large agglomerates, the superposition is less satisfactory for $\mathrm{T}-\mathrm{T}_{\mathrm{g}}{ }^{\omega}<60 \mathrm{~K}$. This can probably be related to the fact - in this last case - that the polymer situated in some of the smallest gaps between particles may strongly contribute to the sample elastic modulus. This idea is coherent with the fact that the reinforcement $\mathrm{R}$ is increasing extremely rapidly with $\mathrm{Tg}_{\mathrm{g}}{ }^{\omega} / \mathrm{T}-\mathrm{T}_{\mathrm{g}}{ }^{\omega}$. For these samples, the volume fraction of the glassy shell is too small to be detected by N.M.R., but the presence of agglomerates amplifies deeply the surface effect. Thus, the gradient of glass transition temperature has - for macroscopic sample - a consequence that depends both on its amplitude but also on the spatial arrangement of the particles.

\section{Conclusion}

The reinforcement of the filled elastomers depends both on temperature and frequency revealing that the dynamics of polymer chains is modified near the particle surfaces. We have analysed this temperature-frequency behavior in the frame of a variation of the glass transition temperature near the particle surface. We have deduced from a model predicting the existence of a glass transition gradient a new temperature-frequency superposition law validated by experiments. The agreement between experimental results and theoretical predictions shows that the temperature and frequency dependence observed on filled elastomers is related to a glass transition temperature gradient in the vicinity of the particles. Its impact on the mechanical properties - for instance the absolute values of the reinforcement - is highly sensitive to the dispersion state. This last effect will be discussed in future papers. 


\section{References and Notes}

[1] Nielsen, L. E. and Landel, R. F. Mechanical Properties of Polymers and Composites, , Marcel Dekker, New York, 1994.

[2] Harwood, J. A. C., Mullins, L. and Payne A.R., J. Appl. Polym. Sci. 1965, 9 , 3011

[3] Krauss, G., J. Appl. Polym. Sci. Appl. Polym. Symp. 1984, 39, 75

[4] Edwards, D.C. J. Mater. Sci. 1990, 25, 4175

[5] Wang MJ, Rubber Chemistry and Technology $1998,71,520$, see also the thesis of Clement F., and Lapra A., Université Paris VI, Paris, France, 1999.

[6] Kaufmann S, Slichter WP, Davis DD, Jour. Polymer. Sci. A2, 1971, 9, 829 .

[7] Struik, L.C.E., Physical Aging in Amorphous Polymers and other Materials. Elsevier, Amsterdam 1978.

[8] Struik, L.C.E., Polymer ,1987, 28, 1521.

[9] Haidar B., Vidal A. Papirer E., Proceeding, Eurofillers 97, Manchester (UK) September 8th -11th, 1997, 239.; Haidar B, Salah Deradji H. Vidal A., Papirer E., Macromol. Symp. 1996, 108, 147.

[10] Tsagaropoulos, G.; Eisenberg, A. Macromolecules 1995, 28, 396 ; Kim, J.S. Jackman; R.; Eisenberg, A. Macromolecules 1994, 272789.

[11] Landry, C.J.T.; Coltrain, B. K.; Landry, M.R.; Fitsgerald, J. J.; Long, V. K. Macromolecules 1993, 26, 3702.

[12] Wallace, W.E., Van Zanten, J.H. and Wu W.L., Phys. Rev. E. 1995, 52, R3329.

[13] Van Zanten, J.H., Wallace, W.E. and Wu W.L. Phys.Rev. E. 1996, 3, R2053.

[14] Keddie, J.L., Jones R.A.L. and Cory R.A. Europhys. Lett. 1994, 27, 59-64.

[15] Forrest, J.L., Dalnoki-Veress, K and Dutcher J.R. Phys. Rev. E. 1997, 56, 5705.

[16] Fukao K., Miyamoto Y. Phys. Rev. E 2000, 61, 1743 
[17] Grohens, Y., Brogly, M., Labbe, C., David, M.O. and Schulzt, J. Langmuir 1998, 14, 2929.

[18] Kawana, S. and Jones, R.A.L. Phys. Rev. E. 2001, 63, 021501-1.

[19] Forrest, JA. And Mattson, J. Phys. Rev. E. 2000, 61, R53.

[20] Long, D. and Lequeux, F. EPJ E. 2001, 4, 371.

[21] Berriot J., Lequeux F., Monnerie L., Montes H., Long D., Sotta P., J of Non Cryst. Solids. 2002, 310,719; Berriot J., Montes H., Sotta P., Polymer. 2002 (submitted)

[22] Jethmalani, J.M. and Ford, W.T., Chem. Mater. 1996, 8, 2138.

[23] Stober W., Fink A., Bohn E., J. Colloid Interf. Sci 1968, 26, 62.

[24] Berriot J., Montes H., Monnerie L., Martin F., Pyckhout-Hintzen W., Meier G., Frielinghaus H. Polymer, 2002 (submitted).

[25] Ediger, M.D. Annu. Rev. Chem. 2000, 51, 99.

[26] Fischer E.W., Hellma G.P., Spiess H.W., Hörth F.J., Ecarius U., Wehrle M. Makromol. Chem. Suppl. $1985,12,89$.

[27] Ferry J. D., Viscoelastic properties of polymers, $3^{\text {rd }}$ Edition, Wiley and SONS, INC, p. 343.

[28] Joseph R., Zhang S., Ford W.T. Macromolecules, 1996,29,1305

[29] Berriot, J.; Montes, H.; Lequeux, F.; Pernot, H. Polymer. 2002, 43 ,6131. 